\title{
ESTUDO COMPARATIVO DE MÉTODOS DE PREPARO DE AMOSTRAS DE TINTA PARA A DETERMINAÇÃO DE METAIS E METALÓIDES POR TÉCNICAS DE ESPECTROMETRIA ATÔMICA
}

\author{
Fabrina R. S. Bentlin, Dirce Pozebon* e Fernanda dos Santos Depoi \\ Instituto de Química, Universidade Federal do Rio Grande do Sul, Av. Bento Gonçalves, 9500, 91509-900 Porto Alegre - RS, \\ Brasil
}

Recebido em 2/4/08; aceito em 25/11/08; publicado na web em 28/4/09

\begin{abstract}
PAINT SAMPLES PREPARATION METHODS FOR METALS AND METHALOIDS DETERMINATION BY ATOMIC SPECTROMETRY TECHNIQUES. This work deals with paint decomposition methods for major, minor and trace elements determination. Three methods were investigated: (1) decomposition in closed quartz vessel and heating in microwave oven; (2) decomposition in open vessel using $\mathrm{HNO}_{3}$ and ashing, following the ASTM D 3335-85a method; and (3) decomposition in open vessel using $\mathrm{HNO}_{3}+\mathrm{HF}$ and ashing. Paints of different types and colours were analyzed, in which several elements were determined using inductively coupled plasma mass spectrometry (ICP-MS) and inductively coupled plasma optical emission spectrometry (ICP OES). It was observed that method (1) is appropriate for trace, minor and major elements determination, while method (3) is appropriate for Ti.
\end{abstract}

Keywords: paints; elements determination; plasma techniques.

\section{INTRODUÇÃO}

A preocupação quanto a elementos tóxicos presentes em tintas, utilizadas para diversas finalidades, é crescente. Isto veio à tona em 2007, quando se viu o recolhimento de brinquedos infantis em função do excesso de $\mathrm{Pb}$ na tinta utilizada no acabamento dos mesmos. $\mathrm{O}$ $\mathrm{Pb}$ apresenta toxidez acumulativa e, uma vez ingerido ou inalado, permanece por longo tempo no organismo causando problemas neurológicos. ${ }^{1}$

A tinta é formada basicamente por resinas, aditivos, solventes e pigmentos. ${ }^{2}$ Os pigmentos são partículas sólidas em forma de pó, constituídas por compostos orgânicos ou inorgânicos, de diferentes cores, ou fluorescentes. Os pigmentos conferem cor e poder de cobertura à tinta, aumentam a proteção e durabilidade da pintura, diminuindo o impacto dos fatores corrosivos da atmosfera, como radiação ultravioleta, umidade e gases corrosivos, afetando a viscosidade, o escoamento, a durabilidade, resistência e outras propriedades físicas.

Os pigmentos orgânicos apresentam em sua estrutura química grupamentos cromóforos (AZO, nitroso, nitro, entre outros) e auxocromos (hidroxila, aminas etc.). Dentre os pigmentos orgânicos mais utilizados estão as ftalocianinas, os monoazóicos, diazóicos e dioxazina. ${ }^{3}$ Os pigmentos inorgânicos são geralmente constituídos por óxidos, cromatos e sulfatos de metais, muitos deles tóxicos $(\mathrm{Pb}, \mathrm{Cr}$, $\mathrm{Co}, \mathrm{Sb}, \mathrm{Cd}$ etc.), conferindo diferentes tonalidades de cor às tintas, conforme a concentração dos pigmentos. ${ }^{3}$ Os pigmentos orgânicos dão mais brilho à tinta e a tornam menos densa. Porém, conferem menor resistência à energia luminosa, a cor é menos estável e a tinta tem menor poder de cobertura e maior custo.

Tintas com altos teores de $\mathrm{Pb}$ são ainda utilizadas em países da Ásia. ${ }^{4}$ No Brasil, foi aprovado em 2007 um projeto de lei que fixa o limite máximo de $\mathrm{Pb}$ em $0,06 \%$, em tintas para uso imobiliário, produtos infantis e material escolar, vernizes e materiais similares de revestimento de superfícies, determinado em base seca ou conteúdo total não-volátil. ${ }^{5}$ Não há ainda legislação para outros elementos.

\footnotetext{
*e-mail : dircepoz@iq.ufrgs.br
}

A matriz da tinta é complexa e de difícil decomposição, tendo-se em vista a presença de diversos compostos orgânicos e inorgânicos. Além disso, os sais que permanecem na solução da amostra que foi decomposta podem interferir na determinação do analito. Os métodos descritos nas normas técnicas existentes restringem-se à determinação de somente alguns elementos $\left(\mathrm{Cd}, \mathrm{Co}, \mathrm{Cr}, \mathrm{Pb}\right.$ e Ti).$^{6-10}$ Nesse sentido, o estudo e desenvolvimento de novas metodologias de determinação de metais e metalóides em tinta são muito importantes.

São encontrados poucos trabalhos envolvendo a determinação de elementos em tintas ${ }^{11-19}$ e a maioria diz respeito à determinação de $\mathrm{Pb} .{ }^{9-14} \mathrm{Em}$ quase todos os trabalhos o estudo envolveu um único tipo de tinta. Os métodos de preparação da amostra geralmente envolveram decomposição parcial da tinta e posterior calcinação, ou decomposição da tinta com mistura de ácidos em forno de microondas. As técnicas de medição mais utilizadas foram a espectrometria de absorção em chama (FAAS) ${ }^{11-13}$ e a espectrometria de emissão óptica com plasma indutivamente acoplado (ICP OES). ${ }^{15-18}$ Foi encontrado apenas um trabalho reportando a utilização da espectrometria de massa com plasma indutivamente acoplado (ICP-MS). ${ }^{14} \mathrm{O}$ trabalho mais recente envolveu a determinação de $\mathrm{Cd}, \mathrm{Co}, \mathrm{Cr}, \mathrm{Cu}, \mathrm{Ni}$ e $\mathrm{Pb}$ em tintas para serigrafia e para tatuagem, por espectrometria de absorção atômica com forno de grafite e amostragem direta de sólidos (DS-GF AAS). ${ }^{19}$ Os resultados obtidos foram validados mediante comparação com GF AAS e ICPMS. Mediante a DS-GF AAS, a tinta pode ser analisada diretamente, bastando apenas a homogeneização da amostra. Contudo, a quantidade de tinta que pode ser introduzida no atomizador é limitada a alguns mg e, conseqüentemente, a técnica é somente adequada para a determinação de concentrações muito baixas de elementos na amostra.

A maioria dos métodos oficiais, publicados na American Society For Testing Materials (ASTM), para a determinação de $\mathrm{Pb}, \mathrm{Cd}$, Co e $\mathrm{Cr}\left[0,01\right.$ a $5 \%(\mathrm{~m} / \mathrm{m})$ de $\mathrm{Pb}, 50$ a $150 \mu \mathrm{g} \mathrm{g}^{-1} \mathrm{de} \mathrm{Cd}, 50$ a $2000 \mu \mathrm{g} \mathrm{g}^{-1} \mathrm{de}$ Co e 0,005 a $1,0 \%(\mathrm{~m} / \mathrm{m})$ de $\mathrm{Cr}$ ] em tintas, recomendam a utilização da F AAS. ${ }^{6,7}$ Contudo, elementos presentes na tinta a nível de $\mu \mathrm{g} \mathrm{g}{ }^{-1}$ a $\%$ podem ser determinados por ICP OES e, além disso, a faixa linear da curva de calibração é ampla e a técnica é de caráter multielementar. Assim sendo, a técnica de ICP OES é bastante adequada para a quantificação de elementos em tinta. Concentrações relativamente baixas de diversos elementos podem ser determinadas por ICP OES, 
principalmente quando a emissão da radiação do analito é focada axialmente. ${ }^{19,20}$ Porém, a sensibilidade pode ser ainda insuficiente para a quantificação de elementos traço presentes em tintas. Neste caso, a técnica de ICP-MS, ${ }^{22,23}$ apesar das mais pronunciadas interferências físicas (supressão de sinal, deposição de material sobre os orifícios de amostragem e flutuações do plasma devido a solventes orgânicos, entre outros), seria mais adequada, pois permite a quantificação de concentrações mais baixas.

O objetivo do presente trabalho é comparar métodos de decomposição de tintas de diferentes tipos, visando a determinação de elementos traço, minoritários e majoritários. Haja vista a complexidade da matriz das amostras e a inexistência de materiais de referência para a maioria dos elementos investigados, três métodos de decomposição são testados, para verificar a exatidão e a precisão dos resultados obtidos. Os elementos traço são determinados por ICP-MS e os minoritários e majoritários por ICP OES.

\section{PARTE EXPERIMENTAL}

\section{Instrumentação}

A determinação dos elementos majoritários e minoritários foi realizada utilizando-se um espectrômetro de ICP OES modelo Optima ${ }^{\mathrm{TM}}$ 2000 DV, da PerkinElmer. Argônio comercial (White Martins/Praxair) foi utilizado para a geração do plasma, como gás de nebulização e auxiliar. Como gás de purga do sistema óptico do equipamento de ICP OES foi utilizado nitrogênio com pureza de 99,996\%, da White Martins/Praxair. Os parâmetros instrumentais usados estão listados na Tabela 1.

Para a determinação dos elementos traço foi utilizado um espectrômetro de massa com plasma indutivamente acoplado (ICP-MS) da PerkinElemer/Sciex (modelo Elan ${ }^{\circledR}$ DRC II), cujos parâmetros principais estão listados na Tabela 1. Nas determinações por ICP OES os sinais das seguintes linhas espectrais (em nm) foram medidos: $\mathrm{Al}$ (I) $(396,153)$, $\mathrm{Ba}$ (II) $(233,527), \mathrm{Ca}(\mathrm{II})(317,933), \mathrm{Cr}(\mathrm{II})(267,716), \mathrm{Cu}(\mathrm{I})(327,393)$, $\operatorname{Mg}(\mathrm{I})(258,213), \mathrm{Pb}(\mathrm{II})(220,353), \operatorname{Sr}(\mathrm{II})(407,771), \mathrm{Ti}(\mathrm{II})(334,940) \mathrm{e}$ Zn(II) (206,200); nas determinações por ICP-MS foram medidos os sinais dos isótopos ${ }^{75} \mathrm{As},{ }^{111} \mathrm{Cd},{ }^{60} \mathrm{Co},{ }^{53} \mathrm{Cr},{ }^{65} \mathrm{Cu},{ }^{55} \mathrm{Mn},{ }^{60} \mathrm{Ni},{ }^{208} \mathrm{~Pb},{ }^{121} \mathrm{Sb},{ }^{120} \mathrm{Sn}$ $\mathrm{e}^{51} \mathrm{~V}$. Linhas espectrais (de todos os analitos) e isótopos adicionais (de $\mathrm{Cd}, \mathrm{Cr}, \mathrm{Ni}, \mathrm{Pb}, \mathrm{Sb}$ e $\mathrm{Sn}$ ) foram monitorados com a finalidade de verificar possíveis interferências espectrais e isobáricas.

Para a decomposição das amostras em sistema fechado foi utilizado forno de microondas (modelo Multiwave $3000^{\circledR}$ da Anton Paar) equipado com rotor específico e frascos de quartzo. O programa de aquecimento utilizado é mostrado na Tabela 2.

\section{Materiais e reagentes}

Foram utilizados $\mathrm{HNO}_{3} 65 \%(\mathrm{~m} / \mathrm{m})$ e HF $48 \%(\mathrm{~m} / \mathrm{m})$ de grau

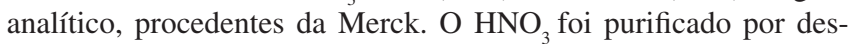
tilação abaixo do ponto de ebulição em um destilador de quartzo (Hans Küner). As soluções foram preparadas com água desionizada com resistividade de $18,2 \mathrm{M} \Omega \mathrm{cm}$, purificada em sistema Milli-Q ${ }^{\circledR}$ da Millipore.

As soluções de calibração foram preparadas em $\mathrm{HNO}_{3} 5 \%(\mathrm{v} / \mathrm{v})$, a partir de diluições adequadas de soluções estoque multielementares: ICP VI da Merck (contendo $10 \mathrm{mg} \mathrm{L}^{-1}$ de $\mathrm{Al}, \mathrm{Ba}, \mathrm{Cd}, \mathrm{Co}, \mathrm{Cr}, \mathrm{Cu}$, $\mathrm{Mg}, \mathrm{Mn}, \mathrm{Ni}, \mathrm{Pb}$ e $\mathrm{Sr} ; 100 \mathrm{mg} \mathrm{L}^{-1}$ de As e $\mathrm{Zn}$ e $1000 \mathrm{mg} \mathrm{L}^{-1}$ de Ca) e CLMS-3 da Spex (contendo $10 \mathrm{mg} \mathrm{L}^{-1}$ de Sb e Sn). Para o Ti foi utilizada solução estoque Titrisol ${ }^{\circledR}$ da Merck, contendo $1000 \mathrm{mg} \mathrm{L}^{-1}$ do elemento. Essas soluções foram utilizadas para a calibração dos instrumentos de ICP-MS e ICP OES. As concentrações das soluções de calibração foram 1-100 $\mu \mathrm{g} \mathrm{L}^{-1}$ para os elementos traço, 100-500 $\mu \mathrm{g}$ $\mathrm{L}^{-1}$ para os minoritários e $500-2000 \mu \mathrm{g} \mathrm{L}^{-1}$ para os majoritários.
Tabela 1. Parâmetros instrumentais usados nas determinações por ICP OES e ICP-MS

\begin{tabular}{lcc}
\hline & ICP OES & ICP-MS \\
\hline $\begin{array}{l}\text { Potência de radiofreqüên- } \\
\text { cia, W }\end{array}$ & 1500 & 1300 \\
$\begin{array}{l}\text { Vazão do gás principal, } \\
\text { L min }{ }^{-1}\end{array}$ & 15 & 15 \\
$\begin{array}{l}\text { Vazão do gás auxiliar, L } \\
\text { min }^{-1}\end{array}$ & 0,2 & 1,2 \\
$\begin{array}{l}\text { Vazão do gás nebulizador, } \\
\text { L min }{ }^{-1}\end{array}$ & 0,8 & 1,16 \\
$\begin{array}{l}\text { Nebulizador } \\
\text { Câmara de nebulização }\end{array}$ & $\begin{array}{c}\text { Ryton }{ }^{\circledR} \text { Scott, } \\
\text { duplo passo }\end{array}$ & Ciclônica \\
$\begin{array}{l}\text { Tubo Injetor } \\
\text { Leituras por replicata }\end{array}$ & $\begin{array}{c}\text { Alumina, } 2 \text { mm de diâmetro interno } \\
\text { Vista de observação }\end{array}$ & Axial e Radial \\
$\begin{array}{l}\text { Cone de amostragem e } \\
\text { skimer }\end{array}$ & - & - \\
Modo de Leitura & - & Pt \\
* para alto teor de sólidos dissolvidos. & peak hopping \\
\hline
\end{tabular}

Tabela 2. Programa de aquecimento do forno de microondas

\begin{tabular}{lcc}
\hline Etapas & Tempo $(\min )$ & Potência $(\mathrm{W})$ \\
\hline Rampa & 5 & $0-700$ \\
Tempo de permanência & 1 & 700 \\
Rampa & 2 & $700-1400$ \\
Tempo de permanência & 20 & 1400 \\
Resfriamento & 20 & 0 \\
\hline
\end{tabular}

Todos os frascos e vidraria utilizados, seja para o armazenamento das amostras ou preparo das soluções de calibração, foram previamente descontaminados em solução de $\mathrm{HNO}_{3} 10 \%$ (v/v), deixando-os em contato com a solução do ácido por $48 \mathrm{~h}$. Após esse período foram lavados com água destilada e desionizada. Os cadinhos de Pt utilizados foram descontaminados com solução de $\mathrm{HCl} 50 \%$ (v/v) sob aquecimento a 90 ${ }^{\circ} \mathrm{C}$ por $6 \mathrm{~h}$. Após este período, os cadinhos foram lavados e enxaguados com água desionizada. Os frascos do forno de microondas também foram devidamente descontaminados, de acordo com o programa de limpeza recomendado pelo fabricante do equipamento.

\section{Amostras}

Foram analisadas tintas de diferentes tipos e cores, para diferentes finalidades, todas fabricadas em 2005 e com validade até 2008, conforme mostrado na Tabela 3.

\section{Preparo das amostras e procedimentos}

Antes da pesagem, as tintas foram manualmente agitadas a fim de ocorrer a homogeneização das mesmas. As amostras de tinta foram decompostas mediante três diferentes métodos (denominados de $\mathrm{M}$, A e F) com exceção das tintas para tatuagem, as quais foram decompostas somente mediante os métodos $\mathrm{M}$ e A. O método $\mathrm{M}$ consistiu na pesagem de 0,200 $\mathrm{g}$ de amostra em frasco de quartzo, ao qual 
foram adicionados $8 \mathrm{~mL}$ de $\mathrm{HNO}_{3}$ concentrado e a mistura aquecida em forno de microondas, conforme o programa mostrado na Tabela 2. Após o devido resfriamento, a solução da amostra foi transferida quantitativamente para frasco de polipropileno graduado e o volume completado a $50 \mathrm{~mL}$ com água. No método A foi seguido procedimento descrito na ASTM D335-85a, ${ }^{6}$ com algumas modificações (a quantidade de amostra utilizada foi reduzida de 2,000 g para 0,200 g e a solução da amostra não foi filtrada). Foram pesados aproximadamente 0,200 g de amostra em um copo Becker, adicionando-se a seguir $5 \mathrm{~mL}$ de $\mathrm{HNO}_{3} 50 \%$ (v/v) e o copo colocado em uma chapa de aquecimento para levar a mistura até a secura, sem ferver. $\mathrm{Na}$ etapa seguinte, o copo com a amostra foi colocado em forno mufla à temperatura ambiente. A seguir, a temperatura do forno foi elevada através de sucessivos incrementos de $50{ }^{\circ} \mathrm{C}$ até $500{ }^{\circ} \mathrm{C}$, permanecendo nesta temperatura por $2 \mathrm{~h}$. A cinza resultante foi solubilizada com 10 $\mathrm{mL}$ de $\mathrm{HNO}_{3} 50 \%$ (v/v), sob aquecimento brando (em torno de 80 ${ }^{\circ} \mathrm{C}$ ) em chapa metálica até a obtenção de um volume final de $5 \mathrm{~mL}$. Essa solução foi transferida quantitativamente para frasco de polipropileno graduado e o volume elevado a $50 \mathrm{~mL}$ com água. $\mathrm{O}$ método $\mathrm{F}$ consistiu em pesar 0,200 g de amostra em cadinho de Pt, adicionar em seguida $3 \mathrm{~mL}$ de $\mathrm{HNO}_{3}$ concentrado e levar a mistura à secura (sem ferver) em chapa de aquecimento. Na etapa seguinte, o cadinho com a amostra foi colocado em forno mufla à temperatura ambiente. A seguir, a temperatura do forno foi elevada através de sucessivos incrementos de $50{ }^{\circ} \mathrm{C}$ até $600{ }^{\circ} \mathrm{C}$, permanecendo nesta temperatura por $2 \mathrm{~h}$. A cinza resultante foi solubilizada com $2 \mathrm{~mL}$ de $\mathrm{HF}$ e $4 \mathrm{~mL}$ de $\mathrm{HNO}_{3}$, sob aquecimento brando (em torno de $80{ }^{\circ} \mathrm{C}$ ) em chapa metálica, até obtenção de um volume final de $3 \mathrm{~mL}$. Essa solução foi transferida quantitativamente para frasco de polipropileno e o volume elevado a $50 \mathrm{~mL}$ com água. Para a determinação dos elementos traço, por ICP-MS, as soluções das amostras foram diluídas 50 vezes com solução de $\mathrm{HNO}_{3} 5 \%$ (v/v). Para a determinação dos elementos majoritários, por ICP OES, as soluções das amostras foram também diluídas com $\mathrm{HNO}_{3} 5 \%$ (v/v), variando-se a diluição de acordo com a concentração do analito na amostra. O conteúdo de material volátil nas tintas foi determinado por gravimetria, utilizando-se cadinho de Pt e aquecimento a $105^{\circ} \mathrm{C}$ por $1 \mathrm{~h}$ em forno mufla.

\section{Análise estatística}

Os resultados obtidos mediante os diferentes métodos foram comparados pela análise de variância (ANOVA) dentro de um intervalo de confiança de $95 \%(\mathrm{p}<0,05)$. Quando as médias foram significativamente diferentes, fez-se o teste de múltipla comparação de Tukey. O programa computacional SPSS, Statistical Package of the Social Sciences, versão $10.0,{ }^{24}$ foi usado para fazer a análise estatística. Além da ANOVA e do teste de Tukey, foi aplicado o teste $t$ (de student), que compara as médias de duas medidas.

\section{RESULTADOS E DISCUSSÃO}

\section{Determinação dos elementos minoritários e majoritários}

Devido à indisponibilidade de tintas contendo concentrações certificadas de metais e/ou metalóides, foram investigados diferentes métodos para decompor as amostras, para melhor avaliar a precisão e exatidão dos resultados obtidos. As concentrações dos elementos minoritários e majoritários, que foram determinadas por ICP OES, são mostradas nas Tabelas 4 e $1 \mathrm{~S}$ (material suplementar). As concentrações de $\mathrm{Cr}, \mathrm{Cu}$, e Pb, não informadas nessas tabelas, não foram determinadas por ICP OES por serem muito baixas, mas o foram por ICP-MS e são mostradas nas Figuras $1 \mathrm{~S}$ e $2 \mathrm{~S}$ (material suplementar).

Nas Tabelas 4 e 1S pode-se notar que as concentrações dos elementos majoritários (expressas em \%), determinadas mediante os três métodos de decomposição são, de maneira geral, concordantes. Com base nos resultados obtidos no teste ANOVA, verificou-se

Tabela 3. Características das tintas analisadas

\begin{tabular}{|c|c|c|c|c|c|}
\hline Tipo e finalidade & Cor & $\begin{array}{l}\text { Solúvel } \\
\text { em água }\end{array}$ & $\begin{array}{l}\text { Conteúdo } \\
\text { volátil, \% }\end{array}$ & $\begin{array}{c}\text { Data de } \\
\text { fabricação }\end{array}$ & $\begin{array}{l}\text { Data de } \\
\text { validade }\end{array}$ \\
\hline \multirow{6}{*}{ Serigrafia } & Branca & não & 42,4 & $10 / 2005$ & $10 / 2008$ \\
\hline & Preta & não & 39,6 & $10 / 2005$ & $10 / 2008$ \\
\hline & Azul & não & 41,6 & $10 / 2005$ & $10 / 2008$ \\
\hline & Vermelha & não & 58,9 & $10 / 2005$ & $10 / 2008$ \\
\hline & Amarela & não & 45,7 & $10 / 2005$ & $10 / 2008$ \\
\hline & Verde & não & 41,9 & $10 / 2005$ & $10 / 2008$ \\
\hline
\end{tabular}

Acrílica - para pinturas artísticas em madeira

Roxa

$\operatorname{sim}$

35,0

$11 / 2005$

$11 / 2008$

\begin{tabular}{|c|c|c|c|c|c|}
\hline \multirow{3}{*}{ Esmalte - uso imobiliário } & Branca & não & 43,0 & $05 / 2005$ & $05 / 2008$ \\
\hline & Verde & não & 50,6 & $05 / 2005$ & $05 / 2008$ \\
\hline & Amarela & não & 45,3 & $05 / 2005$ & $05 / 2008$ \\
\hline \multirow{4}{*}{ Tatuagem } & Preta & $\operatorname{sim}$ & 65,3 & $11 / 2005$ & $11 / 2008$ \\
\hline & Verde & $\operatorname{sim}$ & 71,3 & $11 / 2005$ & $11 / 2008$ \\
\hline & Vermelha & $\operatorname{sim}$ & 73,4 & $11 / 2005$ & $11 / 2008$ \\
\hline & Amarela & $\operatorname{sim}$ & 86,4 & $11 / 2005$ & $11 / 2008$ \\
\hline
\end{tabular}


que a maioria das médias é significativamente igual, com nível de confiança de $95 \%$. As exceções são o Ti na maioria das tintas (Tabelas 4 e $1 \mathrm{~S}$ ), $\mathrm{Al}, \mathrm{Cr}$ e $\mathrm{Pb}$ na tinta esmalte amarela (Tabela $1 \mathrm{~S}$ ), $\mathrm{Pb}$ nas tintas vermelha e amarela para serigrafia e $\mathrm{Mg}$ na tinta amarela, também para serigrafia (Tabela 4). As concentrações de $\mathrm{Al}, \mathrm{Cr}, \mathrm{Pb}$ e Mg determinadas mediante decomposição da amostra em sistema aberto (métodos A e F) são menores e significativamente diferentes daquelas obtidas mediante utilização de sistema fechado (método M). Possivelmente houve perdas dos analitos durante a queima, pois as concentrações determinadas mediante os dois procedimentos em sistema aberto foram concordantes entre si, de acordo com o teste de Tukey. Em relação ao Ti, o método F (utilização de HF) pareceu ser o mais adequado, uma vez que a concentração do elemento determinada na maioria das amostras é mais alta, e significativamente diferente daquelas obtidas mediante os métodos $\mathrm{M} \mathrm{e} \mathrm{A.} \mathrm{Isto} \mathrm{também}$ foi evidenciado no trabalho publicado por Paudyn et al., ${ }^{16}$ o qual cita que foram encontradas concentrações mais baixas de Ti na amostra de tinta decomposta sem adição de HF. É sabido que o HF solubiliza o $\mathrm{TiO}_{2}$ mediante a formação de complexos solúveis e que a presença de Ti nas tintas decorre da adição desse óxido, um dos principais pigmentos brancos utilizados. $\mathrm{O} \mathrm{TiO}_{2}$ é um sólido cristalino, existindo em três formas fundamentais: rutilo tetragonal, prisma tetragonal ou anatase e bruquita ortorômbica (esta é menos importante, não sendo comercialmente produzida). Os cristais de rutilo apresentam uma estrutura mais compacta que os cristais anatase, implicando em grandes diferenças entre as duas formas. O rutilo é mais denso, mais estável e tem um índice de refração maior (este dá maior poder de opacidade à tinta, sendo por isso preferencialmente utilizado em relação ao anatase). ${ }^{2}$ Korn et al. observaram que para a decomposição completa do rutilo é necessário adicionar $\mathrm{HF}^{25}$ Conforme as Tabelas 4 e $1 \mathrm{~S}$, na maioria das tintas analisadas a concentração de Ti encontrada é maior quando o HF é utilizado. As exceções são a tinta amarela para serigrafia (onde as concentrações de Ti encontradas mediante os três métodos são concordantes) e a tinta amarela esmalte (para esta os resultados obtidos mediante os métodos $\mathrm{M}$ e F são concordantes). É provável que nestas duas tintas o anatase tenha sido adicionado, o qual é mais facilmente decomposto, mesmo na ausência de HF. Além disso, como as tintas são diferentes, outros equilíbrios químicos envolvidos podem contribuir para a solubilidade.

Em relação aos elementos minoritários (concentrações em $\mu \mathrm{g}$ $\mathrm{g}^{-1}$ ), observa-se nas Tabelas 4 e $1 \mathrm{~S}$ que as concentrações de $\mathrm{Pb}$ na tinta azul, de $\mathrm{Ca}$ nas tintas branca e preta, todas para serigrafia, e de $\mathrm{Cu}$ na tinta esmalte verde são maiores nas amostras decompostas em sistema aberto (métodos A e F). Os resultados encontrados mediante as decomposições em sistema aberto são significativamente diferentes daqueles encontrados mediante decomposição em sistema fechado (método M). Nesse caso, presume-se que houve contaminação das amostras durante a etapa de decomposição em sistema aberto, uma vez que $\mathrm{Ca}, \mathrm{Cu}$ e $\mathrm{Pb}$ são elementos geralmente presentes no ambiente. Salienta-se que quando a concentração do analito é baixa, a contaminação pode influenciar significativamente no resultado final. As concentrações de $\mathrm{Mg}$, Sr e $\mathrm{Zn}$ determinadas através dos procedimentos de decomposição em sistema fechado (método $\mathrm{M}$ ) e decomposição com $\mathrm{HNO}_{3}$ em copo aberto (método A) são estatisticamente iguais, para a maioria das tintas analisadas (Tabelas 4 e 1S). No entanto, a concentração de Ba determinada na tinta acrílica roxa (Tabela $1 \mathrm{~S}$ ) mediante o método $\mathrm{F}$ (as cinzas foram solubilizadas com $\mathrm{HNO}_{3}$ e HF) é estatisticamente diferente das encontradas mediante os demais métodos. A tinta acrílica roxa contém teores mais altos de $\mathrm{Ca}$ e $\mathrm{Zn}$ e, de acordo com a Tabela $3 \mathrm{~S}$, estes parecem ter sido parcialmente perdidos mediante adição de HF, eliminando parcialmente a matriz. De fato, observou-se a existência de material insolúvel quando o HF não foi adicionado e é possível que parte do Ba tenha ficado ocluído no precipitado. A máxima potência do plasma foi utilizada $(1500 \mathrm{~W})$, mas devido ao curto tempo de residência no plasma, é possível que o material insolúvel contendo Ba ocluído não tenha sido decomposto na sua totalidade, impedindo a atomização, ionização e excitação de todo o Ba presente. Pode-se observar ainda na Tabela 4 que as concentrações de $\mathrm{Al}, \mathrm{Ca}, \mathrm{Mg}$ e $\mathrm{Sr}$ na tinta verde e de $\mathrm{Ca}, \mathrm{Cr}, \mathrm{Mg}$ e $\mathrm{Sr}$ na tinta amarela, ambas para serigrafia, são menores mediante a adição de HF (método F). Pela ANOVA, as concentrações médias de Al, $\mathrm{Ca}, \mathrm{Mg}$ e $\mathrm{Sr}$ relacionadas com o método $\mathrm{F}$ são significativamente diferentes. Perdas dos analitos durante a queima a $600{ }^{\circ} \mathrm{C}$, ou sob a forma de fluoreto durante a solubilização das cinzas são possíveis causas para as concentrações mais baixas encontradas.

Na tinta verde esmalte foi encontrada concentração relativamente baixa de $\mathrm{Cu}$ (Tabela 3S), o qual, quando presente na forma de ftalocianina, confere cor verde às tintas. No entanto, a concentração de Fe (não mostrada nas Tabelas 4 e 1S) determinada na tinta verde esmalte é $0,23 \%(\mathrm{~m} / \mathrm{m})$, e a combinação do azul de ferro $\left[\mathrm{Fe}\left(\mathrm{NH}_{4}\right) \mathrm{Fe}(\mathrm{CN})_{6}\right] \mathrm{com}$ o amarelo de cromo origina o verde de cromo, provável responsável pela cor verde da tinta em questão. Dependendo da relação amarelo de cromo e azul de ferro obtém-se cor verde de diferentes tonalidades, sendo que a forma cristalina e o tamanho das partículas são fatores que também influenciam na tonalidade da cor. $\mathrm{O}$ amarelo de cromo (possivelmente como $\mathrm{PbCrO}_{4} \times \mathrm{PbSO}_{4}$, amarelo claro) estaria presente em baixa concentração na tinta verde esmalte, tendo-se em vista os baixos teores de $\mathrm{Cr}(0,02 \% \mathrm{~m} / \mathrm{m})$ e $\mathrm{Pb}(0,07 \% \mathrm{~m} / \mathrm{m})$ encontrados. ${ }^{2}$

Quanto às tintas para tatuagem (os resultados da análise destas tintas não são mostrados nas Tabelas 4 e 3S), foram determinados por ICP OES apenas o Ca em todas as tintas (a concentração variou de 95 a $\left.358 \mu \mathrm{g} \mathrm{g}^{-1}\right), \mathrm{Cu}$ na tinta verde $(1,57 \% \mathrm{~m} / \mathrm{m})$, Zn na tinta preta $(0,78 \% \mathrm{~m} / \mathrm{m})$ e na tinta vermelha $\left(23,3 \mu \mathrm{g} \mathrm{g} \mathrm{g}^{-1}\right)$. As concentrações de $\mathrm{Ca}, \mathrm{Cu}$ e $\mathrm{Zn}$ encontradas mediante os métodos $\mathrm{A}$ e $\mathrm{M}$ foram significativamente iguais ao nível de confiança de 95\% (de acordo com o teste $t$ ). Como a maioria dos elementos investigados nas tintas para tatuagem era menor que o LD (limite de detecção) o método F não foi testado para essas tintas. Baixas concentrações de vários elementos determinados em tintas para tatuagem são reportadas no trabalho de Bentlin et al. ${ }^{19}$ No entanto, conforme acima citado, foram encontradas altas concentrações de $\mathrm{Cu}$ numa amostra e de $\mathrm{Zn}$ em outra.

Nas Tabelas 4 e $1 \mathrm{~S}$ pode-se observar que em algumas tintas (vermelha, amarela e verde para serigrafia e amarela esmalte) a concentração de $\mathrm{Pb}$ é alta (até $14 \% \mathrm{~m} / \mathrm{m}$ ), demonstrando que foram usados pigmentos inorgânicos à base de $\mathrm{Pb}$ para a formulação dessas tintas, com prazo de validade até outubro de 2008 (Tabela 3).

A partir dos resultados encontrados para os elementos majoritários e minoritários, com exceção do Ti, pode-se concluir que o método de decomposição em forno de microondas pode ser recomendado para a decomposição de tintas. No entanto, devem ser empregados frascos de quartzo, por causa da mais alta temperatura atingida. Vale salientar que no presente trabalho foi observada apenas decomposição parcial da tinta quando utilizados copos de Teflon®, ao invés de copos de quartzo. Dependendo da técnica de medição do analito, a água régia pode ser usada para auxiliar na decomposição. No presente estudo a água régia não foi testada, tendo-se em vista as interferências espectrais causadas, principalmente pelo $\mathrm{Cl}$ na determinação de As e Mn por ICP-MS.

\section{Determinação dos elementos traço}

Os elementos presentes em concentrações mais baixas nas tintas (elementos traço) foram determinados por ICP-MS, cujos resultados encontrados são mostrados nas Figuras 1, 1S e 2S (material suplementar). 
Na Figura 1 observa-se que as concentrações de $\mathrm{Co}$ e $\mathrm{Cu}$ na maioria das tintas analisadas, $\mathrm{Cr}$ na tinta azul e $\mathrm{Cd}$ na vermelha, ambas para serigrafia, são semelhantes; as concentrações médias determinadas mediante os três métodos não têm diferença significativa, com nível de confiança de $95 \%$. Entretanto, a concentração de $\mathrm{Pb}$ determinada nas tintas branca e azul, ambas para serigrafia (Figura 1), Ni na tinta verde esmalte e Mn em quase todas as tintas analisadas (Figuras 1, 1S e 2S) são maiores e estatisticamente diferentes quando a amostra é decomposta em sistema aberto. Além disso, as concentrações determinadas mediante os dois procedimentos em sistema aberto são concordantes entre si (de acordo com o teste de Tukey). A contaminação que ocorre em sistema aberto pode ser uma das causas da concordância, uma vez que também foi observada contaminação das provas em branco. Além disso, há menos resíduos da matriz nas soluções obtidas mediante calcinação, diminuindo as interferências nas determinações por ICPMS. Mas, estudos adicionais devem ser feitos para garantir que as concentrações mais altas de Mn encontradas são devidas à melhor decomposição da amostra mediante calcinação.

As concentrações de $\mathrm{Sb}$ e $\mathrm{Sn}$, determinadas mediante os três métodos de decomposição, não foram concordantes para a maioria das amostras analisadas (Figuras 1 e 1S). Concluiu-se que as metodologias de decomposição utilizadas não são apropriadas para a determinação de $\mathrm{Sb}$ e $\mathrm{Sn}$ em tintas. Esses elementos são voláteis e mais facilmente perdidos em sistema aberto e, além disso,

Tabela 4. Concentrações (em $\% \mathrm{~m} / \mathrm{m}$, em base seca) de elementos majoritários e minoritários nas tintas para uso em serigrafia, determinados por ICP OES. M: decomposição com $\mathrm{HNO}_{3}$ em copo de quartzo fechado e com aquecimento em forno de microondas; A: queima em copo

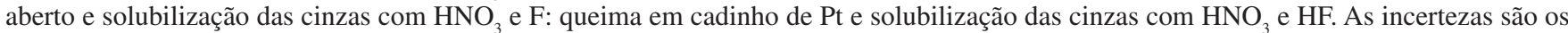
desvios padrão de três determinações. As concentrações de $\mathrm{Cr}, \mathrm{Cu}$ e Pb não informadas foram determinadas por ICP-MS e são mostradas na Figura 1S (material suplementar)

\begin{tabular}{|c|c|c|c|c|c|c|c|}
\hline \multirow{2}{*}{ Elemento } & \multirow{2}{*}{ Método } & \multicolumn{5}{|c|}{ Cores } & \multirow[b]{2}{*}{ Verde } \\
\hline & & Branca & Preta & Azul & Vermelha & Amarela & \\
\hline \multirow[t]{3}{*}{$\mathrm{Al}$} & M & $0,54 \pm 0,02$ & $265 \pm 66^{*}$ & $0,22 \pm 0,02$ & $0,13 \pm 0,01$ & $0,26 \pm 0,02$ & $0,15 \pm 0,01$ \\
\hline & A & $0,52 \pm 0,02$ & $147 \pm 45^{*}$ & $0,21 \pm 0,02$ & $0,11 \pm 0,02$ & $0,24 \pm 0,02$ & $0,14 \pm 0,01$ \\
\hline & $\mathrm{F}$ & $0,45 \pm 0,07$ & $391 \pm 17^{*}$ & $0,21 \pm 0,03$ & $0,13 \pm 0,01$ & $0,22 \pm 0,02$ & $0,09 \pm 0,004$ \\
\hline \multirow[t]{3}{*}{$\mathrm{Ba}$} & M & $4,69 \pm 0,73$ & $<0,2^{*}$ & $<0,2^{*}$ & $0,51 \pm 0,08$ & $0,74 \pm 0,04$ & $0,21 \pm 0,02$ \\
\hline & A & $4,26 \pm 0,36$ & $<0,9^{*}$ & $<0,9^{*}$ & $0,59 \pm 0,05$ & $0,75 \pm 0,02$ & $0,19 \pm 0,02$ \\
\hline & $\mathrm{F}$ & $3,87 \pm 0,75$ & $<0,3^{*}$ & $<0,3^{*}$ & $0,68 \pm 0,08$ & $0,70 \pm 0,04$ & $0,19 \pm 0,09$ \\
\hline \multirow[t]{3}{*}{$\mathrm{Ca}$} & M & $358 \pm 21^{*}$ & $98 \pm 10^{*}$ & $72 \pm 22^{*}$ & $0,14 \pm 0,002$ & $13,8 \pm 0,2$ & $16,9 \pm 0,5$ \\
\hline & A & $412 \pm 23^{*}$ & $129 \pm 5^{*}$ & $106 \pm 10^{*}$ & $0,11 \pm 0,01$ & $13,8 \pm 0,4$ & $16,6 \pm 0,4$ \\
\hline & $\mathrm{F}$ & $370 \pm 16^{*}$ & $121 \pm 3^{*}$ & $96 \pm 2^{*}$ & $0,13 \pm 0,006$ & $9,9 \pm 0,6$ & $11,6 \pm 0,9$ \\
\hline \multirow[t]{3}{*}{$\mathrm{Cr}$} & M & - & - & - & $1,90 \pm 0,19$ & $1,07 \pm 0,04$ & $0,76 \pm 0,03$ \\
\hline & A & - & - & - & $1,61 \pm 0,24$ & $1,03 \pm 0,02$ & $0,76 \pm 0,03$ \\
\hline & $\mathrm{F}$ & - & - & - & $1,73 \pm 0,07$ & $0,83 \pm 0,11$ & $0,71 \pm 0,05$ \\
\hline \multirow[t]{3}{*}{$\mathrm{Cu}$} & M & - & - & $0,79 \pm 0,12$ & - & - & $0,43 \pm 0,02$ \\
\hline & A & - & - & $0,70 \pm 0,10$ & - & - & $0,40 \pm 0,12$ \\
\hline & $\mathrm{F}$ & - & - & $0,80 \pm 0,12$ & - & - & $0,38 \pm 0,03$ \\
\hline \multirow[t]{3}{*}{$\mathrm{Mg}$} & M & $377 \pm 3^{*}$ & $43 \pm 10^{*}$ & $60 \pm 2^{*}$ & $88 \pm 3^{*}$ & $0,75 \pm 0,04$ & $684 \pm 3^{*}$ \\
\hline & A & $384 \pm 5^{*}$ & $41 \pm 7^{*}$ & $55 \pm 2 *$ & $90 \pm 2^{*}$ & $0,50 \pm 0,13$ & $646 \pm 50^{*}$ \\
\hline & $\mathrm{F}$ & $330 \pm 19^{*}$ & $60 \pm 2^{*}$ & $31 \pm 3^{*}$ & $58 \pm 8^{*}$ & $0,20 \pm 0,17$ & $594 \pm 40^{*}$ \\
\hline \multirow[t]{3}{*}{$\mathrm{Pb}$} & M & - & - & $46 \pm 3^{*}$ & $9,50 \pm 0,07$ & $4,97 \pm 0,02$ & $4,34 \pm 0,02$ \\
\hline & A & - & - & $139 \pm 50^{*}$ & $8,31 \pm 0,71$ & $4,82 \pm 0,07$ & $4,15 \pm 0,12$ \\
\hline & $\mathrm{F}$ & - & - & $113 \pm 34^{*}$ & $9,24 \pm 0,36$ & $3,96 \pm 0,61$ & $3,96 \pm 0,26$ \\
\hline \multirow[t]{3}{*}{$\mathrm{Sr}$} & M & $775 \pm 9^{*}$ & $<0,02^{*}$ & $<0,02^{*}$ & $371 \pm 10^{*}$ & $687 \pm 2^{*}$ & $928 \pm 14^{*}$ \\
\hline & A & $589 \pm 45^{*}$ & $<0,8^{*}$ & $<0,8^{*}$ & $359 \pm 17^{*}$ & $685 \pm 9^{*}$ & $878 \pm 43^{*}$ \\
\hline & $\mathrm{F}$ & $509 \pm 59^{*}$ & $<0,01^{*}$ & $<0,01^{*}$ & $490 \pm 34^{*}$ & $606 \pm 37^{*}$ & $789 \pm 52^{*}$ \\
\hline \multirow[t]{3}{*}{$\mathrm{Ti}$} & M & $7,66 \pm 0,23$ & $<0,3^{*}$ & $3,27 \pm 0,02$ & $31 \pm 2^{*}$ & $0,48 \pm 0,02$ & $195 \pm 24^{*}$ \\
\hline & A & $2,85 \pm 0,38$ & $<0,7^{*}$ & $2,79 \pm 0,24$ & $29 \pm 8^{*}$ & $0,39 \pm 0,09$ & $255 \pm 77^{*}$ \\
\hline & $\mathrm{F}$ & $16,0 \pm 0,71$ & $<0,5^{*}$ & $5,05 \pm 0,36$ & $68 \pm 12^{*}$ & $0,52 \pm 0,22$ & $637 \pm 57^{*}$ \\
\hline \multirow[t]{3}{*}{$\mathrm{Zn}$} & M & $2,33 \pm 0,03^{*}$ & $0,73 \pm 0,05^{*}$ & $8,14 \pm 0,38^{*}$ & $5,29 \pm 0,68^{*}$ & $52 \pm 6^{*}$ & $45 \pm 5^{*}$ \\
\hline & A & $<3,2^{*}$ & $<3,2^{*}$ & $<3,2^{*}$ & $7,65 \pm 1,78^{*}$ & $42 \pm 4^{*}$ & $41 \pm 2^{*}$ \\
\hline & $\mathrm{F}$ & $<0,3^{*}$ & $<0,3^{*}$ & $<0,3^{*}$ & $<0,3^{*}$ & $39 \pm 7^{*}$ & $40 \pm 3^{*}$ \\
\hline
\end{tabular}

: concentrações em $\mu \mathrm{g} \mathrm{g}^{-1}$; os valores precedidos por $<$ são os limites de detecção do método. 

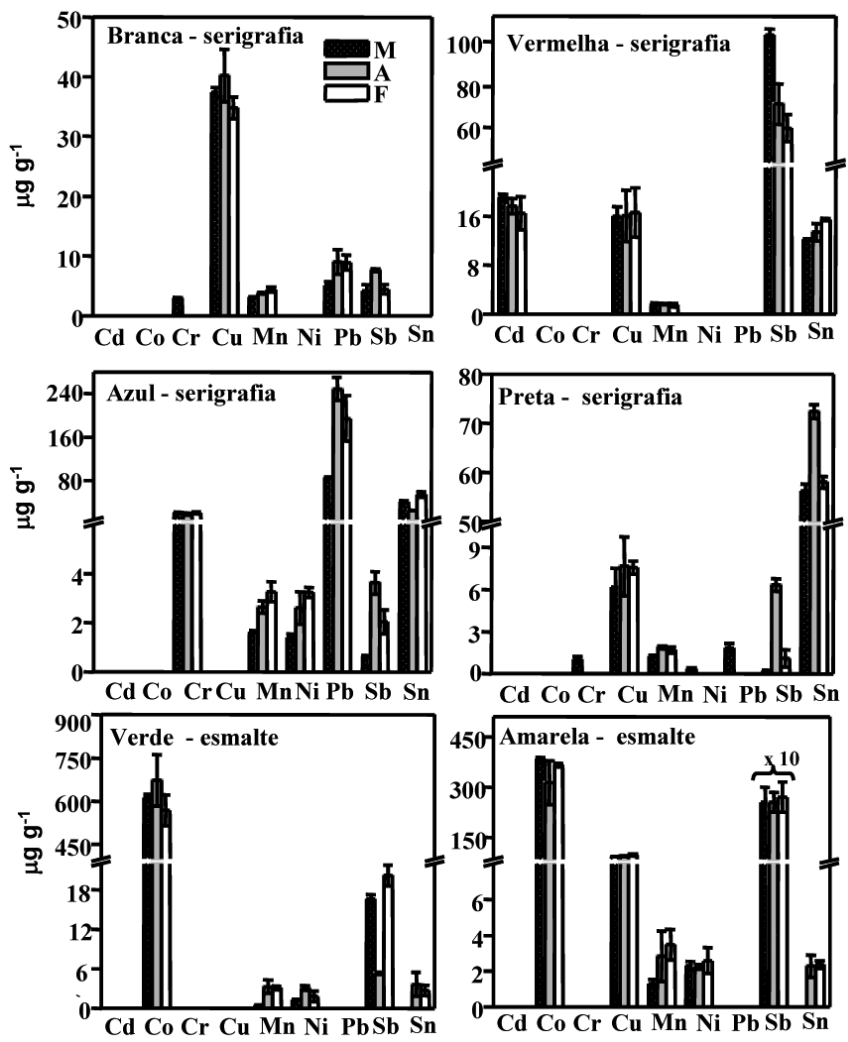

Figura 1. Concentrações dos elementos determinadas por ICP-MS nas tintas. M: decomposição em copo de quartzo com aquecimento em forno de microondas; A: decomposição com $\mathrm{HNO}_{3}$ e calcinação em copo aberto e F: decomposição com $\mathrm{HF}+\mathrm{HNO}_{3}$ e calcinação em cadinho de Pt. Os resultados são expressos em $\mu g^{-1}$ e as barras de erro representam o desvio padrão de três determinações. A ausência de barras indica que os elementos não foram detectados na amostra

precipitam em meio contendo $\mathrm{HNO}_{3}$, o qual é recomendado para melhor decompor a tinta. Nota-se ainda que a concentração de $\mathrm{Sb}$ é relativamente alta na tinta amarela esmalte (Figura 1), indicando que pode ter havido adição de $\mathrm{Sb}$ e, segundo Paudin et al.,${ }^{16}$ o óxido de antimônio tem sido utilizado em tintas. Na Figura 1 observa-se também que as concentrações de $\mathrm{Ni}$ e $\mathrm{Pb}$ na tinta preta e $\mathrm{Cr}$ nas tintas branca e preta, todas para serigrafia, não foram detectadas nas amostras decompostas em sistema aberto, devido a possíveis perdas dos analitos. Isso também pode ser observado para o As e $\mathrm{V}$ em todas as tintas analisadas (Figura $1 \mathrm{~S}$ ).

Em relação às tintas para tatuagem (Figura $2 \mathrm{~S}$ ) as concentrações determinadas mediante os métodos $\mathrm{M}$ e $\mathrm{A}$ foram concordantes, a um nível de confiança de $95 \%$ (teste $t$ ). Porém, as concentrações de Mn (nas tintas verde e vermelha) obtidas mediante o método A (sistema aberto) foram mais elevadas, o que pode também ser observado para outras tintas cujas concentrações são mostradas na Figura 1. As concentrações dos elementos que não aparecem nas Figuras 1 , $1 \mathrm{~S}$ e $2 \mathrm{~S}$ não foram detectadas, cujos LDs variaram de 0,002 a $0,03 \mu \mathrm{g} \mathrm{g}^{-1}$.

\section{CONCLUSÕES}

Com base nos resultados obtidos para os diferentes métodos testados, pode-se concluir que o método de decomposição da tinta em copo de quartzo fechado com aquecimento em forno de microondas é o mais adequado, com exceção do Ti. Para este elemento, o método de decomposição com HF em cadinho de Pt e aquecimento convencional mostrou-se mais adequado.
Comparando-se as concentrações determinadas mediante os três métodos, maiores diferenças foram encontradas em relação aos elementos traço, possivelmente por causa da contaminação que ocorre em sistema aberto. Contudo, mesmo com a utilização de copos de quartzo fechados e aquecimento com microondas, como acima citado, observou-se que a decomposição de algumas tintas é melhor mediante calcinação. Mas, quando este procedimento é usado, alguns elementos são perdidos, principalmente As e V.

Através do presente trabalho foi evidenciada a necessidade de desenvolver e padronizar métodos de decomposição de tintas, principalmente quando se objetiva a determinação de elementos traço e minoritários, levando-se em conta o tipo de tinta e o elemento a ser determinado.

As concentrações de $\mathrm{Pb}$ encontradas em algumas tintas para uso em serigrafia (vermelha, verde e amarela) são mais altas que o limite permitido para o $\mathrm{Pb}(0,06 \% \mathrm{~m} / \mathrm{m})$, reportado na Lei $\mathrm{N}^{\circ} 5.334 / 2005$. $^{5}$ Acredita-se que o teor de $\mathrm{Pb}$ nas tintas agora produzidas seja menor, uma vez que a lei foi recentemente aprovada. Contudo, elementos tóxicos como Co e Sb também foram encontrados em algumas tintas.

\section{MATERIAL SUPLEMENTAR}

No material suplementar disponível em http://quimicanova. sbq.org.br, na forma de arquivo PDF, com acesso livre e gratuito, encontram-se figuras e tabela referentes às concentrações dos elementos determinados nas tintas, por ICP OES (Tabela 1S) e ICP-MS (Figuras $1 \mathrm{~S}$ e 2S).

\section{AGRADECIMENTOS}

À CAPES pela bolsa de mestrado de F. R. S. Bentlin e ao Setor de Química Industrial e Ambiental do Departamento de Química da UFSM pelo empréstimo do forno de microondas e equipamento de ICP-MS.

\section{REFERÊNCIAS}

1. Kunert, H. J.; Wielmüller, G. A.; Röbbecke, R. S.; Ebel, H.; Küppers, M. M.; Podoll, K.; Int. J. Hyg. Environ. Health 2004, 207, 521.

2. Fazenda, J. M. R.; Tintas e Vernizes: Ciência e Tecnologia, $2^{\mathrm{a}}$ ed., Abrafati: São Paulo, 1995.

3. Saron, C.; Felisberti, M. L.; Quim. Nova 2006, 29, 124.

4. Clark, C. S.; Rampal, K. G.; Truppil, V.; Chen, C. K.; Clark, R.; Roda, S.; Environ. Res. 2006, 102, 9.

5. Diário Oficial da União; Lei n. 5.334, Brasília, 6 de fevereiro de 2007, p. 569.

6. American Society for Testing Materials. ASTM D 3335-85a; Standard Test Method for Low Concentrations of Lead, Cadmium and Cobalt in Paint by Atomic Absorption Spectroscopy, 2005.

7. American Society for Testing Materials. ASTM D 3718-85a; Standard Test Method for Low Concentrations of Chromium in Paint by Atomic Absorption Spectroscopy, 2005.

8. American Society for Testing Materials. ASTM D 4764-01; Determination by X-ray Fluorescence Spectroscopy of Titanium Dioxide Content in Paint, 2006.

9. American Society for Testing Materials. ASTM D 3618; Standard Test Method for Detection of Lead in Paint and Dried Paint Films, 1999.

10. International Organization for Standardization. ISO 6503; Paints and Varnishes - Determination of total lead - Flame atomic absorption spectrometric method, 1984.

11. Hausknecht, K. A.; Ryan, E. A.; Leonard, L. P.; At. Spectrosc. 1982, 3, 53.

12. Corl, W. E.; At. Spectrosc. 1991, 6, 40.

13. Pimenta, A. S.; Vital, B. R.; Nunes, G. S.; Jordão, C. P.; Quim. Nova 1994, 17, 227. 
14. Viczián, M.; Lásztity, A.; Barnes, R. M.; J. Anal. At. Spectrom. 1990, $5,293$.

15. Guerrero, M.; Todoli, J. L.; Anal. Lett. 1999, 32, 771.

16. Paudyn, A. M.; Smith, R. G.; Fresenius J. Anal. Chem. 1993, 345, 695.

17. Paudyn, A. M.; Smith, R. G.; J. Anal. At. Spectrom. 1990, 5, 523.

18. Sadiq, M.; Zaidi, T. H.; Sheikheidin, S. Y.; Bull. Environ. Contam. Toxicol. 1995, 54, 451.

19. Bentlin, F. R. S.; Pozebon, D.; Mello, P. A.; Flores, E. M. M.; Anal. Chim. Acta. 2007, 602, 23.

20. Petry, C. F.; Pozebon, D.; Bentlin, F. R. S.; At. Spectrosc. 2005, $26,19$.
21. Petry, C. F.; Dissertação de Mestrado, Universidade Federal do Rio Grande do Sul, Brasil, 2005.

22. Becker, J. S.; Inorganic Mass Spectrometry: principles and applications; $1^{\text {st }}$ ed., John Wiley \& Sons Ltd: Chichester, 2007.

23. Pozebon, D.; Tese de Doutorado, Universidade Federal de Santa Catarina, Brasil, 1998.

24. MICROSOFT, SPSS Base 10.0 Application Guide, Canadá, 1999.

25. Korn, M. G.; Ferreira, A. C.; Costa, A. C. S.; Nóbrega, J. A.; Silva, C. R.; Microchem. J. 2002, 71, 41. 
ESTUDO COMPARATIVO DE MÉTODOS DE PREPARO DE AMOSTRAS DE TINTA PARA A DETERMINAÇÃO DE METAIS E METALÓIDES POR TÉCNICAS DE ESPECTROMETRIA ATÔMICA

Fabrina R. S. Bentlin, Dirce Pozebon* e Fernanda dos Santos Depoi

Instituto de Química, Universidade Federal do Rio Grande do Sul, Av. Bento Gonçalves, 9500, 91509-900 Porto Alegre - RS, Brasil
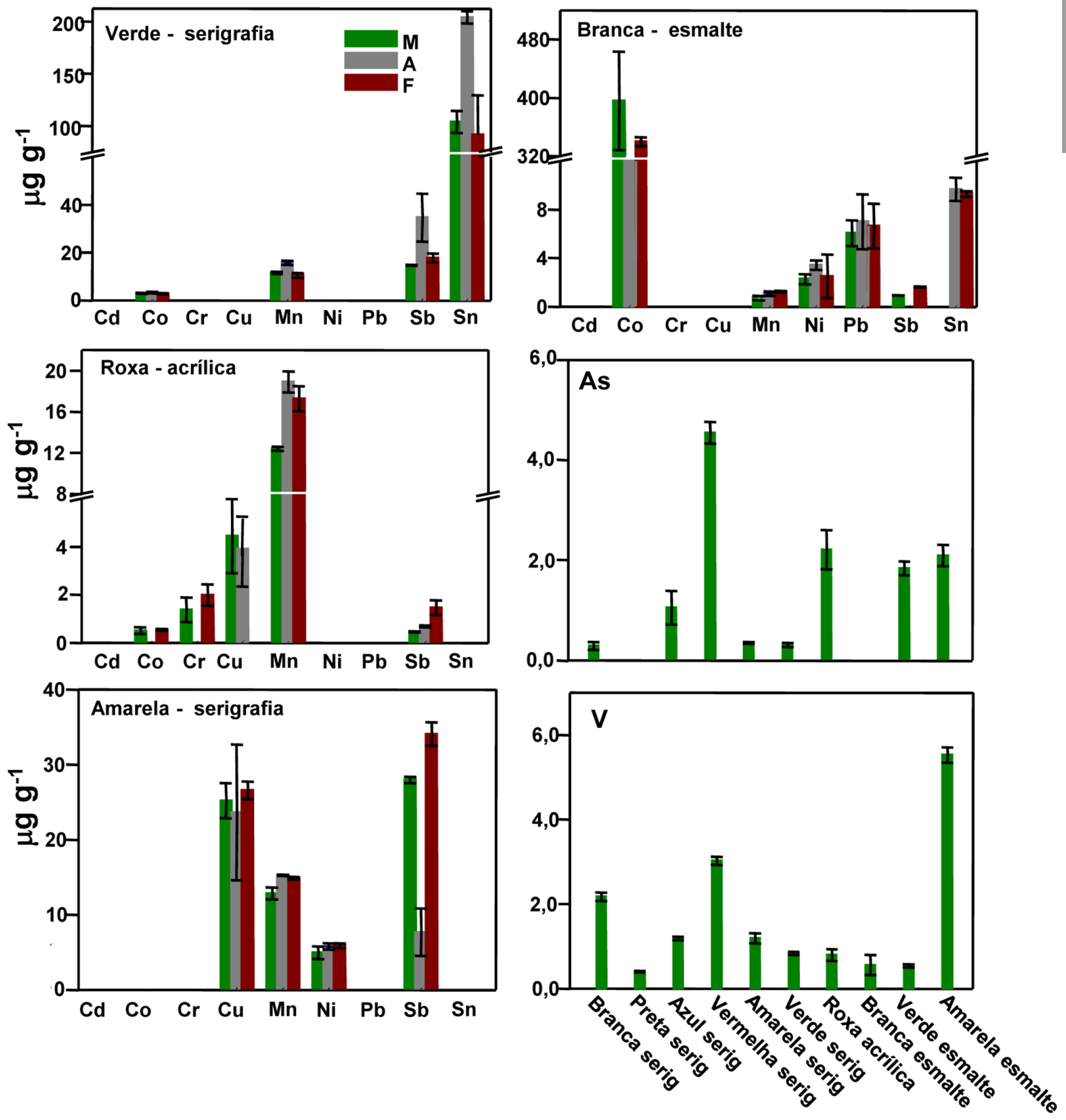

Figura 1S. Concentrações dos elementos determinadas por ICP-MS nas tintas para serigrafia e esmalte. M: decomposição em copo de quartzo com aquecimento em forno de microondas; A: decomposição com $\mathrm{HNO}_{3}$ e calcinação em copo aberto e $\mathrm{F}$ : decomposição com $\mathrm{HF}+\mathrm{HNO}{ }_{3} e$

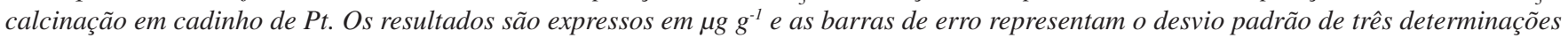
(análise em triplicata) 

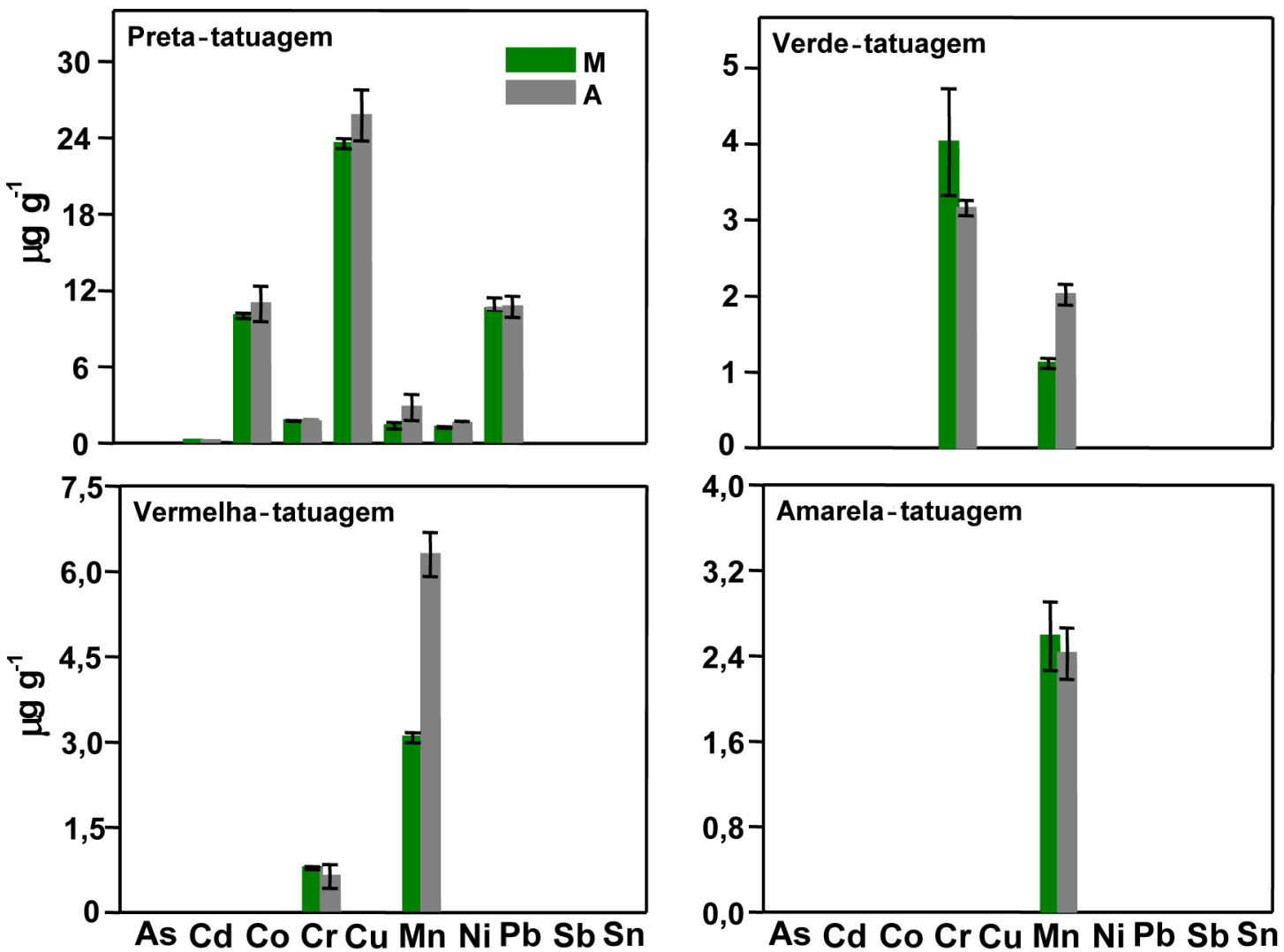

Figura 2S. Concentrações dos elementos determinadas por ICP-MS em tintas para tatuagem; M: decomposição em copo de quartzo com aquecimento em forno de microondas e A: decomposição com $\mathrm{HNO}_{3}$ e calcinação em copo aberto. Os resultados são expressos em $\mu \mathrm{g} \mathrm{g}^{-1}$ e as barras de erro representam o desvio padrão de três determinações (análise em triplicata) 
Tabela 1S. Concentrações (em \% m/m, em base seca) de elementos majoritários e minoritários nas tintas, determinados por ICP OES. M: decomposição com $\mathrm{HNO}_{3}$ em copo de quartzo fechado e com aquecimento em forno de microondas; A: queima em copo aberto e solubilização das cinzas com $\mathrm{HNO}_{3}$ e F: queima em cadinho de Pt e solubilização das cinzas com $\mathrm{HNO}_{3}$ e HF. As incertezas são os desvios padrão de três determinações. As concentrações de $\mathrm{Cr}, \mathrm{Cu}$ e $\mathrm{Pb}$ não informadas foram determinadas por ICP-MS e são informadas na Figura $1 \mathrm{~S}$

\begin{tabular}{|c|c|c|c|c|c|}
\hline \multirow{3}{*}{ Elemento } & \multirow{3}{*}{ Método } & \multicolumn{4}{|c|}{ Tintas } \\
\hline & & Roxa & Branca & Verde & Amarela \\
\hline & & Acrílica & Esmalte & Esmalte & Esmalte \\
\hline \multirow[t]{3}{*}{$\mathrm{Al}$} & M & $0,23 \pm 0,02$ & $0,42 \pm 0,02$ & $1300 \pm 8^{*}$ & $0,20 \pm 0,02$ \\
\hline & A & $0,20 \pm 0,02$ & $0,46 \pm 0,07$ & $460 \pm 38^{*}$ & $0,13 \pm 0,02$ \\
\hline & $\mathrm{F}$ & $0,120 \pm 0,02$ & $0,37 \pm 0,11$ & $439 \pm 43^{*}$ & $0,13 \pm 0,02$ \\
\hline \multirow[t]{3}{*}{$\mathrm{Ba}$} & M & $16,9 \pm 0,8^{*}$ & $125 \pm 54^{*}$ & $81 \pm 2^{*}$ & $0,37 \pm 0,04$ \\
\hline & A & $12,3 \pm 0,3^{*}$ & $121 \pm 5^{*}$ & $77 \pm 4^{*}$ & $0,33 \pm 0,02$ \\
\hline & $\mathrm{F}$ & $35,4 \pm 1,1^{*}$ & $112 \pm 11^{\mathrm{a}}$ & $91 \pm 41^{*}$ & $0,27 \pm 0,05$ \\
\hline \multirow[t]{3}{*}{$\mathrm{Ca}$} & M & $16,2 \pm 0,2$ & $114 \pm 18^{*}$ & $443 \pm 10^{*}$ & $177 \pm 24^{*}$ \\
\hline & A & $16,6 \pm 1,4$ & $132 \pm 33^{*}$ & $217 \pm 21^{*}$ & $221 \pm 33^{*}$ \\
\hline & $\mathrm{F}$ & $15,7 \pm 0,5$ & $130 \pm 4^{*}$ & $328 \pm 24^{*}$ & $201 \pm 9^{*}$ \\
\hline \multirow[t]{3}{*}{$\mathrm{Cr}$} & M & - & - & $217 \pm 2^{*}$ & $2,56 \pm 0,05$ \\
\hline & A & - & - & $221 \pm 10^{*}$ & $1,61 \pm 0,42$ \\
\hline & $\mathrm{F}$ & - & - & $207 \pm 6^{*}$ & $2,07 \pm 0,05$ \\
\hline \multirow[t]{3}{*}{$\mathrm{Cu}$} & M & - & - & $176 \pm 6^{*}$ & - \\
\hline & A & - & - & $190 \pm 4^{*}$ & - \\
\hline & $\mathrm{F}$ & - & - & $211 \pm 16^{*}$ & - \\
\hline \multirow[t]{3}{*}{$\mathrm{Mg}$} & M & $0,10 \pm 0,001$ & $47 \pm 7^{*}$ & $152 \pm 6^{*}$ & $46 \pm 2^{*}$ \\
\hline & A & $0,10 \pm 0,006$ & $42 \pm 4^{*}$ & $63 \pm 24^{*}$ & $53 \pm 15^{*}$ \\
\hline & $\mathrm{F}$ & $0,09 \pm 0,004$ & $<0,6^{*}$ & $65 \pm 8^{*}$ & $42 \pm 4^{*}$ \\
\hline \multirow[t]{3}{*}{$\mathrm{Pb}$} & M & - & - & $798 \pm 12^{*}$ & $14,3 \pm 0,02$ \\
\hline & A & - & - & $733 \pm 8^{*}$ & $12,4 \pm 0,04$ \\
\hline & $\mathrm{F}$ & - & - & $719 \pm 89^{*}$ & $12,1 \pm 0,26$ \\
\hline \multirow[t]{3}{*}{$\mathrm{Sr}$} & M & $0,10 \pm 0,001$ & $0,14 \pm 0,003$ & $0,20 \pm 0,02$ & $0,16 \pm 0,001$ \\
\hline & A & $0,10 \pm 0,004$ & $0,17 \pm 0,003$ & $0,22 \pm 0,02$ & $0,16 \pm 0,006$ \\
\hline & $\mathrm{F}$ & $0,10 \pm 0,001$ & $0,16 \pm 0,005$ & $0,24 \pm 0,02$ & $0,17 \pm 0,006$ \\
\hline \multirow[t]{3}{*}{$\mathrm{Ti}$} & M & $3,43 \pm 0,14$ & $4,44 \pm 0,04$ & $0,57 \pm 0,06$ & $0,73 \pm 0,07$ \\
\hline & A & $3,79 \pm 0,02$ & $4,12 \pm 0,05$ & $0,30 \pm 0,02$ & $0,18 \pm 0,05$ \\
\hline & $\mathrm{F}$ & $5,81 \pm 0,22$ & $14,1 \pm 0,6$ & $1,52 \pm 0,12$ & $0,79 \pm 0,07$ \\
\hline \multirow[t]{3}{*}{$\mathrm{Zn}$} & M & $12,0 \pm 2,5^{*}$ & $0,14 \pm 0,01$ & $0,16 \pm 0,003$ & $0,14 \pm 0,01$ \\
\hline & A & $11,9 \pm 0,9^{*}$ & $0,12 \pm 0,001$ & $0,13 \pm 0,01$ & $0,14 \pm 0,01$ \\
\hline & $\mathrm{F}$ & $7,0 \pm 1,3^{*}$ & $0,11 \pm 0,01$ & $0,12 \pm 0,008$ & $0,13 \pm 0,01$ \\
\hline
\end{tabular}

*: concentrações em $\mu \mathrm{g} \mathrm{g}^{-1}$; os valores precedidos por < são os limites de detecção do método. 